\title{
Relational Optimization and Its Application: From Bottleneck Flow Control to Wireless Channel Allocation
}

\author{
Mario KÖPPEN \\ Network Design and Research Center \\ Kyushu Institute of Technology \\ 680-4 Kawazu, Iizuka, Fukuoka 820-8502, Japan \\ e-mail:mkoeppen@ieee.org
}

Received: September 2011; accepted: March 2013

\begin{abstract}
Relational mathematics, as it is studied in fields like mathematical economics and social choice theory for some time, provides a rich and general framework and appears to be a natural and direct way to paraphrase optimization goals, to represent user preferences, to justify fairness criterions, to cope with QoS or to valuate utility. Here, we will focus on the specific application aspects of formal relations in network design and control problems and provide the general concept of relational optimization. In relational optimization, we represent the optimization problem by a formal relation, and the solution by the set of maximal (or non-dominated) elements of this relation. This appears to be a natural extension of standard optimization, and covers other notions of optimality as well. Along with this, we will provide a set of fairness relations that can serve as maximizing relations in relational optimization according to various application needs, and we specify a meta-heuristic approach derived from evolutionary multi-objective optimization algorithms to approximate their maximum sets.
\end{abstract}

Key words: relational mathematics, relational optimization, binary relations, optimization.

\section{Introduction}

Recently, advances in network technologies and their increasing impact on all our daily's life have raised a new quality of problems with regard to optimization, efficiency and controllability. The change is essentially coming from the stronger reference to the subjectivity of agents, expressed in terms like preference, equity, fairness, or envy-freeness. As an example, consider a scenario for a Broadband Wireless Access (BWA) system, the so-called OFDMA (Orthogonal Frequency-Division Multiple Access) system, which also employs cooperation among various agents via relays. In such a model, three types of stations (mobile stations, relay stations, base station) are placed in a plane. There is a complex interplay between the stations, but the main goal is to direct downlink traffic from the base station to the mobile stations, partially utilizing the relay stations. There are many influencing factors as well as features of a specific configuration of such a system (see Zhang et al., 2009 for a nice introduction into this problem): 
- the allocation of directing transmissions from mobile stations to relay stations in a cooperative manner, or to the mobile stations directly, and the share of such a cooperation,

- the number and placement of relay stations (probably taking physical terrain conditions or antenna heights into account),

- the power allocation to relay stations with corresponding signal-to-noise ratios, and choices of their forwarding mode: decode-and-forward, where the relay station decodes the transmission from the base station, encodes anew and forwards to the mobile station, causing additional delay, amplify-and-forward, where the relay station just amplifies the base station transmission, resulting into higher power usage, or a mixture of both modes,

- minimum transmission rates, maximum delays, and/or maximum tolerable error rates seeked by the mobile stations,

- subcarrier allocation to stations with corresponding channel coefficients, and the accompanying splitting of transfer rates into low and high rates that influence channel utilization,

- the management of time slots, etc.

There is no immediate specification of an efficient guidance for the system configuration, while exactly this is needed to set up and operate the system at each time instant. Common ways to achieve such a guidance are optimality, fairness, Quality-of-Service (QoS), among many other like inferring from simulation studies or descriptions of probability distributions, identification of equilibrium states etc. We can easily see that in such a context, even the most generic concept among them, i.e. optimization can be understood in various manners, and be given in terms of efficiency, maximality, "better-ness" or robustness. QoS usually refers to features that are guaranteed throughout the operational time of the system, and often appear as constraints to the optimization. These are all referring to objective circumstances. By fairness, for the first time we also introduce subjectivity of agents.

Here, we want to put forward a theory that appeared in a number of recent publications in a coherent manner, which utilizes aspects of economics as efficient guidance on a strict relational framework. Section 2 will recall basic facts about relations and tailor this to the needs of relational optimization, expressed by the task of finding maximum sets of relations. Section 3 will introduce a special class of relations, fairness relations, and its utilization in network design and control problems will be exemplified in Section 4. The paper will draw some conclusions and compare to related work in Section 5.

\section{Relations and Relational Optimization}

In set theory, a binary relation $R$ on a set $X$ is simply a subset of $X \times X$, indicating all (ordered) pairs $(x, y)$ of elements from $X$ between which the relation holds. Thus, $R$ is basically a set

$$
x R y \quad \Leftrightarrow \quad(x, y) \in R \subseteq X \times X,
$$


and we speak about " $x$ is in relation to $y$," "the relation is between $x$ and $y$ " etc. This is a special case of a relation between elements from a set $A$ and elements from a set $B$ (which is a subset of $A \times B$ ) where $A=B$, and it is also a special case of an $n$-ary relation as subset of $n$-times the direct product of $X$ with itself, where $n=2$.

There are several ways to represent a relation, in addition to the set representation. For example, if $X=\{1,3,5\}$, then

$$
X \times X=\{(1,1),(1,3),(1,5),(3,1),(3,3),(3,5),(5,1),(5,3),(5,5)\} .
$$

We consider a "larger"-relation $R_{>}$as the subset of pairs $\{(3,1),(5,1),(5,3)\}$, but it seems to be more transparent to represent it as:

$$
R=\{(x, y) \mid x, y \in X \wedge x>y\}
$$

This is the logical representation of $R$ as a set comprehension, i.e. a statement describing the properties that its member pairs must satisfy. Another way is the functional representation as a mapping $(1 \rightarrow \emptyset, 3 \rightarrow\{1\}, 5 \rightarrow\{1,3\})$ that assigns to each $x \in X$ the subset of elements of $X$ to which it is in relation.

Assuming an ordering of $X$ so that $X$ is composed of elements $x_{i}$ with $i=1, \ldots,|X|$, then

$$
R=R_{i j}=\left(\begin{array}{ccc}
0 & 0 & 0 \\
1 & 0 & 0 \\
1 & 1 & 0
\end{array}\right)
$$

gives a matrix representation of the same relation, where $R_{i j}=1$ if and only if $\left(x_{i}, x_{j}\right) \in R$. Among the many other ways to represent a relation, we only want to mention the graph representation of a relation as a directed graph $G=(V, E)$ of node set $V$ and edge set $E$. In our example, the nodes of the graph are the elements of $X$, and there is an edge from $x \in X$ to $y \in X$ if and only if $(x, y)$ belongs to the relation.

Since the various representations refer to different mathematical concepts, we can "borrow" terms from the corresponding disciplines and use them for relations as well. For example, we can speak about the spanning tree of a relation, or its diameter, according to the graph representation. According to the matrix representation, we can speak about the rank, or the Eigenvalues of a relation etc.

The set $X$ is often called the domain of the relation. Given a relation $R$ between elements of $X$, we can define a restricted relation between elements of any subset of $X$. If $Z \subseteq X$ then $R_{Z}$ is the set of all pairs $(x, y) \in R$ such that $x \in Z$ and $y \in Z$.

From a practical point of view, relations can refer to various things, or, in other words, can have different interpretations. A few examples:

- Relations can represent equivalence of things, or that two things are representing the same. It is usually expressed as = relation. Close to equivalence, there are also the aspects of approximation or similarity $(\approx)$ and of identity $(\equiv)$. 
- Very often, we use the term "relation" with respect to a social relation, like father-of, daughter-of, boss-of etc., thus referring to a position in a hierarchy.

- Feasible spaces of problem domains directly refer to a relation among its elements while usually we will need an $n$-ary relation for its representation.

- Relations can refer to some aspect of "better-ness" like more, better, larger, "at least as good", dominates, is-preferred-to etc. and thus help characterizing a percept from environment.

- Relations can also refer to a temporal or logical order, esp. to represent causality, precedence, or linkage.

- Non-binary relations are also used to represent pairings (like (Apple, Banana) and are of utmost importance for the formal handling of relational databases.

For distinguishing all these different aspects of relations, properties of relations can be considered. Among the basic properties that can be found in any textbook are:

- Completeness: $\forall x, y \in X: x \neq y \rightarrow(x, y) \in R \vee(y, x) \in R$,

- Reflexivity: $\forall x \in X:(x, x) \in R$,

- Irreflexivity: $\forall x \in X:(x, x) \notin R$,

- Antisymmetry: $(x, y) \in R \wedge(y, x) \in R \rightarrow x=y$,

- Transitivity: $\forall x, y, z \in X:(x, y) \in R \wedge(y, z) \in R \rightarrow(x, z) \in R$, or

- Symmetry: $\forall x, y \in X:(x, y) \in R \rightarrow(y, x) \in R$.

There are many specific properties, and we will see a few more later on. Then, classes of relations are specified by composing elementary properties: a relation is an Equivalence, if it is reflexive, transitive and symmetric. Or: a relation is an Ordering, if it is reflexive, antisymmetric and transitive. But as already indicated by re-using the bold font here, these compound properties are just properties as well, and we may logically combine them with other compound properties to represent presumed "elementary" properties as compound properties. This is the far reach of the Ugly Duckling Theorem (Watanabe, 1969), and it also means that there is no way to grasp the semantic meaning of a relation (equivalence, similarity, better-ness etc.) by means of properties alone, i.e. between two such relations the total number of distinguishing properties (all compounds from a set of basic properties) between two differently interpreted relations is constant. It means we have to ponder the properties, in order to come up with a practical approach.

With regard to the set representation of a relation, we can define corresponding operations on relations, in order to get new relations from given ones. A few examples:

- Complement: $(x, y) \in \tilde{R} \leftrightarrow(x, y) \notin R$,

- Converse: $(x, y) \in \hat{R} \leftrightarrow(y, x) \in R$,

- Converse Complement: the converse of the complement of a relation $R$ (same as the complement of the converse),

- Relational Product: $(x, z) \in\left(R_{1} \times R_{2}\right) \leftrightarrow \exists y \in X:(x, y) \in R_{1} \wedge(y, z) \in R_{2}$ ( $R_{1}, R_{2}$ both have the same domain $X$, also note that this operation the base for the definition of relational algebra),

- Transitive Closure: The smallest $R_{T C}$ such that $R \subseteq R_{T C} \subseteq X \times X$ and $R_{T C}$ is transitive, 
- Symmetric Part: $S(R)=\{(x, y) \in R \mid(y, x) \in R\}$, or

- Asymmetric Part: $P(R)=\{(x, y) \in R \mid(y, x) \notin R\}$.

The above-mentioned problems with the specification of relations by properties alone come up when we consider special sets specified by relations. While we can apply the following definitions to any relation, they make only sense if the relations belong to a special class of algorithms. Nevertheless, two of these special sets, maximum set and best set, will be the major focus of the remaining part of this paper.

(a) Upper Approximation of a set $Z \subseteq X$ by a relation $R$ is given as the set $\hat{Z}_{R}=\{x \in$ $X \mid \exists z \in Z:(x, z) \in R\}$. This is referring to the character of the relation as a similarity relation, and appears this way in rough set theory, along with the related definition.

(b) Lower Approximation of $Z \subseteq X$ by $R$ as $\check{Z}_{R}=\{z \in Z \mid \forall x \in X:(x, z) \in R \rightarrow$ $x \in Z\}$.

Per definition, we could also consider upper and lower approximations of sets by order or equivalence relations, but here, they would just coincide with the sets $Z$. Thus, some care has to be taken about the proper meaning of a relation, and this also applies for the following two specifications, where the focus is on the better-ness character of a relation $R$.

(c) Maximum Set $M(R)$ of a relation $R$ is given as the subset of all elements of $X$ to which no other (different) element of $X$ is in relation $P(R)$, formally

$$
x \in M(R) \longleftrightarrow \nexists y \in X, \quad y \neq x:(y, x) \in P(R)
$$

followed by the specification.

(d) Best Set $B(R)$ of a relation $R$ as the set of all elements of $X$ that are in relation $P(R)$ to any other element of $X$, formally:

$$
x \in B(R) \longleftrightarrow \forall y \in X, \quad y \neq x:(x, y) \in P(R) .
$$

Both definitions could be applied to any kind of relation, but also, as long as we are focusing on better-ness, they do not serve an interesting purpose. For example, for a temporal order of events, the maximum set refers to the last event, and the best set to the first event. For an equivalence relation, both sets are empty since the asymmetric part is empty. We say that the maximum set is composed of maximal elements, and the best set is composed of greatest elements. In either case, these are extreme elements.

The reference to the asymmetric part $P(R)$ is for formal convenience. We are basically interested in the relation to non-equivalent elements in order to justify maximality or greatestness. If considering the same definitions based on $R$ instead of $P(R)$, these sets will probably be modified, and we speak about strong maximum sets and weak best sets. It can be seen that maximum and best sets are related concepts: the maximum set of a relation is the same as the weak best set of the converse complement relation, and the best set of a relation is the same as the strong maximum set of the converse complement relation.

We will also use the "infix" notation $x \geqslant_{R} y$ to refer to a relation $R$ between $x$ and $y$ with the interpretation of better-ness, and by $x>_{R} y$ to its asymmetric part $P(\geqslant)$. 


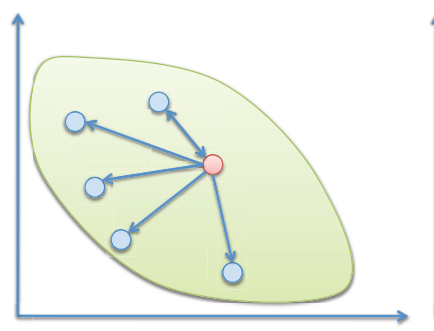

Greatest Element from Best Set

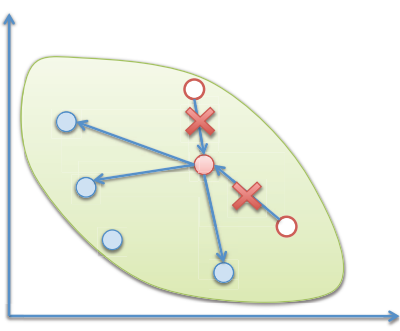

Maximal Element from Maximum Set

Fig. 1. Illustrating maximum and best set elements for a graph representation of a relation. The figures show only relations involving extreme elements.

So far, we have recalled mostly fundamental facts about relations that can be found in many textbooks. But if someone compares the various sources for introducing relations, it can be seen that there are slight variations in the way of defining things - like a "dialect" of speaking about relations. In fact, so far we have followed the "dialect" of a mathematical economist, for a good reason. Relations play a central role in mathematical economics to formalize the concept of social choice, and the strict application of relational mathematics here was provided by Suzumura (2010).

What is a social choice function? Given is a choice set $X$ (for example of goods to distribute) and a system $S$ of subsets $s_{i}$ of the choice set $X$. Then a choice function $C$ assigns to each subset $s_{i}$ of $S$ one of its non-empty subsets $C\left(s_{i}\right)$. This notation can represent many concepts, for example an election: the choice set $X$ are all electable persons of a community, and the subsets $s_{i}$ the sets of candidates for various elections. Then, a voting scheme can be applied to the totality of these candidate sets, comprising the set $S$ with the effect of selecting a winner for each contained candidate set. In this case, the subset of $s_{i}$ of chosen candidates usually contains exactly one element, but in other circumstances, the chosen subset can also have more than one element. Figure 2 illustrates the case where the choice set $X$ has 7 elements. Here, the choice function does not have to provide means for choices from all possible subsets, but only for two subsets: it selects two items for subset $s_{1}$ and one item for subset $s_{2}$ (indicated by open circles). Note that not all elements of $X$ need to belong to a subset, and that elements can also belong to more than one subset.

Then Suzumura introduced the concept of rationalizability of a social choice. In general, this refers to the special case of a social choice function, where we can specify a relation $R$ among the elements of $X$ such that for each element of $S$ the chosen subset $C\left(s_{i}\right)$ of $s_{i}$ corresponds with the best (or maximum) set of the restricted relation $R_{s_{i}}$. For some reason, Suzumura actually defines rationalizability based on what we called weak best set, but the small differences in formal notation will not be relevant for the aspects studied here.

Figure 3 illustrates a rationalization of the choice function that was shown in Fig. 2 by providing a correspondence between choices from subsets and greatest elements (it also 


\section{Choice Function}

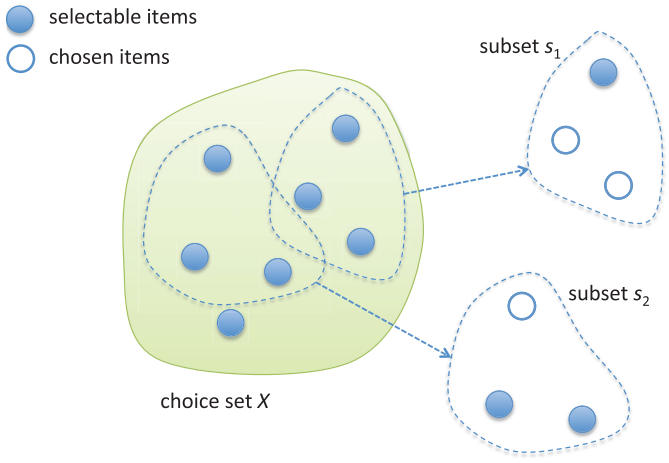

Fig. 2. Social choice from a choice set.

Rationalization

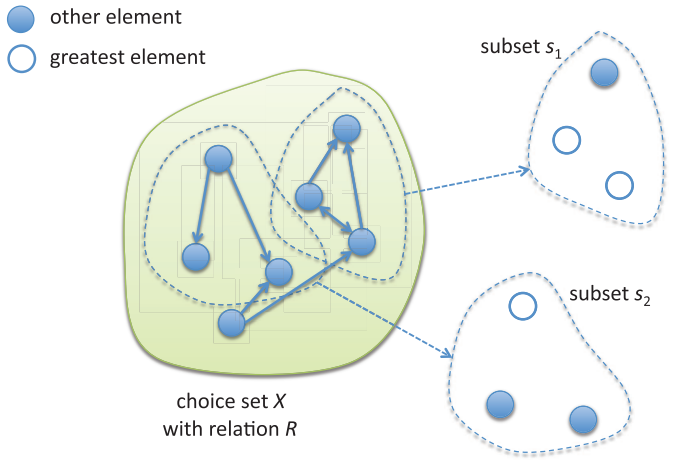

Fig. 3. Rationalizability of a social choice function.

contains a case where a greatest element is not maximal, but chosen nevertheless).

In general, not all choice functions will have a rationalizing relation, and there can be more than one rationalization of the same choice function. We say that a choice function is rationalizable if at least one rationalizing relation exists.

In Suzumura (2010) it is also demonstrated how various economical theories can be brought under the umbrella of this formal framework, by providing additional assumptions about the choice functions as so-called axioms. For example, Arrow's Axiom is given as:

$$
\forall s_{1}, s_{2} \in S: s_{1} \subset s_{2} \rightarrow\left[s_{1} \cap C\left(s_{2}\right)=\emptyset \vee s_{1} \cap C\left(s_{2}\right)=C\left(s_{1}\right)\right]
$$

The "meaning" of this may take a moment to comprehend. It is virtually exclusion of choices depending on absence of choice items. Assume a scenario where someone goes into a cafe and wants to order cake, and the waiter explains the menu choices blueberry cake, cheesecake, and cherry cake. The customer decides for the blueberry cake. After a 

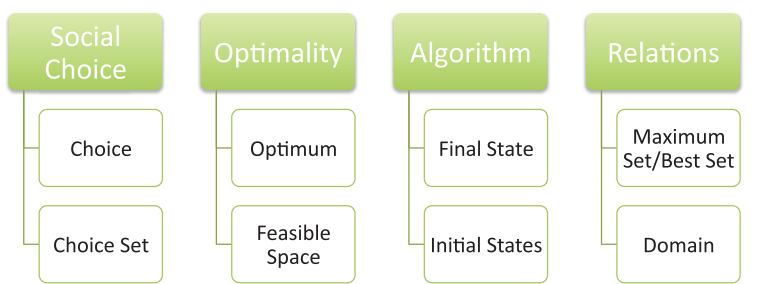

Correspondence

Fig. 4. Universal rationalizability, extending the concept of a social choice function to other domains.

moment, the waiter returns to the table and excuses that he made a mistake when telling the choices, and there is no cheesecake available anymore. The, the customer replies "If that's the case, I will take the cherry cake!" Such a change of mind is actually excluded by Arrow's Axiom for a "rational" agent. There, $s_{2}$ is $\{$ cheese, cherry, blueberry $\}$ and $s_{1}=$ $\{$ cherry, blueberry\}. Then, the customer is the "performer" of the choice function $C$ and this assigns $C\left(s_{1}\right)=\{$ cherry $\}$ and $C\left(s_{2}\right)=\{$ blueberry $\}$. So we have $s_{1} \subset s_{2}$, that means the range of available items $s_{2}$ is narrowed down to $s_{1}$ ("no cheesecake"). But the implication appears to be wrong: $s_{1} \cap C\left(s_{2}\right)=\{$ blueberry $\}$ is not empty, and $s_{1} \cap C\left(s_{2}\right)=\{$ blueberry $\}$ is different from $C\left(s_{1}\right)=\{$ cherry $\}$.

So, Arrow's Axiom reads like: if the range of available items $\left(s_{2}\right)$ is narrowed down to $s_{1}$, but still contains some previously chosen items, no previously unchosen item becomes chosen and no previously chosen item becomes unchosen.

As an example for the further reasoning: based on this assumption about the choice function, and if the choice function is rationalizable by a relation $R$ the relation is an ordering if and only if the choice function fulfills Arrow's Axiom (Theorem 2.2 in Suzumura, 2010).

This is a very short and sketchy introduction to the Suzumura approach to social choice, and we highly recommend the book (Suzumura, 2010) for further reading. As it was stated in the introduction, our concern is optimality as a means to efficiently select configurations for design and operation of a system. However, the pure set notation of rationalizability implies its applicability to various other contexts, and we want to call this universal rationalizability (see Fig. 4). Rationalizability was given by the correspondence between best or maximum sets and social choices. But also if we want to formalize optimality, we refer to the selection of optimal states for each element of a collection of subsets of the feasible space, and we can consider its rationalization by a relation. Then, the optimal states corresponds with the maximum or best sets of the relation, restricted to the corr. subset. In fact, the traditional approach to optimization is to maximize a realvalued function, so the relation here is simply the $>$ relation (alternatively $\geqslant$ ), where 
both, the best and maximum sets correspond with the largest element or supremum. ${ }^{1}$ In multi-objective optimization, we refer to the Pareto-front (i.e. the maximum set of the Pareto-dominance relation) of non-dominated elements in a similar sense (see next section). But the correspondence also shows that we can provide optimality by specifying any other "better-ness" relation in the same fashion. This is the approach of relational optimization.

The other argument is about algorithms. There might be different opinions about the meaning of "algorithm" but it might not cause to much critique if one says that an algorithm is a step-by-step procedure of state transitions, starting from an initial state and arriving at a final state. If we also agree that the final state is just an ordinary member of the possible initial states, the concept of rationalizability applies as well: for some algorithms there might exists a relation such that the selection of a final state coincides with the maximum or best set of a relation among the elements of the initial states. This is another correspondence of universal rationalizability. The last one discussed here is that then, an algorithm can solve an optimization problem, if they share a rationalizing relation. But also, given an algorithm, we can ask what kind of optimality it provides by identifying a fitting relation. This principle will gives us guidance in the following. By using a relation as a rationale, a selection principle can become efficient.

\section{Fairness Relations}

In this section, we will use rationalization of fairness as a means for optimality, which means we will represent fairness as a relations between states of choice. Before doing so, a few comments about the general idea of fairness are in place. Actually, fairness refers to several concepts. In social sciences, three such concepts are: distributive fairness, which is about the modality of a distribution of (dividable or individable) goods; procedural fairness as judgement of a decision making process; and interactional fairness, with regard to the perception of interpersonal treatment (Azar and Darvishi, 2011; Hack and Lammers, 2007). In all cases, we can find an aspect of "empathy" in the judgement about fairness, by one agent virtually taking the position of another one to compare states (a sloppy way of paraphrasing this is the common washing room motto: "Please leave the room the way you would like to find it!"). Thus, if there would be only one agent in the universe, there would be no fairness (as well as no unfairness), but also, the empathies here are not cycling, that means an agent will not take the virtual position of the virtual position of another agent easily. Nevertheless, we use fairness for comparison.

If we want to represent fairness as a relation between states, we can consider some properties of such relations (in an axiomatic sense). This will be introduced in the following:

\footnotetext{
${ }^{1}$ To be formally correct, we have to consider the function as providing a pre-ordering relation between the elements of its domain, and we are looking for the maximum set of this pre-order.
} 
Definition 1. A relation $R$ between elements of a set $X$ is non-cyclic or cycle-free if there is no finite sequence of elements $x_{i}$ with $i=1, \ldots, n$ such that

$$
x_{1} \geqslant_{R} x_{2} \geqslant_{R} x_{3} \geqslant_{R} \cdots \geqslant_{R} x_{n}>_{R} x_{1} .
$$

Following Suzumura, there a various concepts of cyclicity of a relation, according to various needs and with and without reference to the asymmetric part of a relation. In any case, cycle-freeness is a weakened form of transitivity. As mentioned before, with regard to fairness, we may consider one agent justifying by taking the position of another agent, but usually it is hard to take the position of a taken position by a third agent. Therefore, a formal representation of fairness is not necessarily transitive. By cycle-freeness we ensure two things: (1) even if we cannot conclude from $x>_{R} y$ and $y>_{R} z$ that also $x>_{R} z$, but at least we do not have $z>_{R} x$; (2) for finite $X$, the relation will have a non-empty maximum set.

We also need some relational "bounds" to ensure that a fairness relation is still a relation acknowledging global improvements.

For vector relations, where $X \subseteq R^{n}$, we recall Pareto-dominance.

Definition 2. For any pair of vectors $x, y \in R^{n}$ we say that $x$ (weakly) Pareto-dominates $y(x \geqslant p y)$ if and only if for all $i=1, \ldots, n, x_{i} \geqslant y_{i}$.

Note that often there is the additional requirement that for at least one index $j, x_{j}>y_{j}$. This is actually the asymmetric part of the just defined Pareto-dominance, so using the notation $x>_{p} y$ to refer to this variant (also called strong Pareto-dominance) should avoid any confusion. As said, we are not so much interested into equality when looking for extreme elements.

By using Pareto-dominance, we define another property of a vector relation.

Definition 3. A vector relation $R$ between elements of a subset $X$ of $R^{n}$ is called rightPareto-transitive (RPT), if for all $x, y, z \in X$ from $x \geqslant_{R} y$ and $y \geqslant_{p} z$ also follows $x \geqslant_{R} z$.

By RPT we refer to the fact that for $x \geqslant_{R} y$, the aspect is that of $x$ "looking at" $y$ and not the other way. If this is the case, any deterioration of $y$ should preserve the relation. We do not consider the dual property of left-Pareto-transitivity (LPT, $x \geqslant_{p} y \wedge y \geqslant_{R} z \rightarrow$ $x \geqslant_{R} z$ ), as this would result to the rather complex situation to take the viewpoint of an agent $y$ taking the viewpoint of $z$ while changing $x$ at the same time. Nevertheless, we will have relations with this property as well.

As a last comment, we will define antisymmetric relations $\geqslant_{R}$ but practically only focusing on their symmetric part $>_{R}$, to have a clear distinction in any case. Then:

Definition 4. A vector relation between elements of a subset $X$ of $R^{n}$ is a fairness relation if and only if it is antisymmetric, cycle-free, and right-Pareto-transitive.

We hope that foregoing comments hopefully supported the reasoning behind this definition. We will provide some examples for fairness relations. 
(1) As the simplest case, the $\geqslant$-relation between real numbers is a fairness relation. From $a \geqslant b$ and $b \geqslant a$ we conclude that $a=b$. As a transitive relation it is necessarily cycle-free, and also RPT.

(2) Pareto-dominance is a fairness relation as well: it is transitive and antisymmetric, and RPT is just another reading of the transitivity condition.

(3) Any vector relation using a scalar comparison with a monotone scalar function is a fairness relation. Scalar comparison here means to justify whether $x \geqslant_{R} y$ or not by using a (scalar) mapping $f: X \rightarrow R$ and testing $f(x) \geqslant f(y)$. Specific examples here are comparison by the average of components, or any other power means.

(4) Maxmin fairness appears as the characteristic of a stable state in traffic rate allocation in wired networks (Bertsekas and Gallager, 1992). It is given as a state where an agent can only become better off if another agent, already equally or worse off, becomes even more worse off. This gives raise to the following definition of a maxmin fairness relation:

Definition 5. Given a feasible space $X \subseteq R^{n}$. For two elements (vectors) $x$ and $y$ from $X$ it is said that $x$ maxmin fair dominates $y\left(x \geqslant{ }_{m m f} y\right)$ iff for each $i$ with $y_{i}>x_{i}$ there is at least one $j \neq i$ such that (1) $x_{i} \geqslant x_{j}$ and (2) $x_{j}>y_{j}$.

This relation is a little bit hard to comprehend at the beginning. Also from a practical point of view, we may simplify the evaluation whether $x>_{m m f} y$ or not as follows: separate the set $\{1, \ldots, n\}$ of indizes of $x$ into three groups. Group $A$ are all indizes where $x_{i}>y_{i}$, set $B$ all indizes where $x_{i}=y_{i}$, and set $C$ all indizes where $x_{i}<y_{i}$. Then, $x>{ }_{m m f} y$ if and only if $\min (A) \leqslant \min (C){ }^{2}$ We can also say that $A$ are the "looser" (if $x$ takes the $y$-perspective) and $C$ are the "winner" in the comparison. Then, it is considered at least as fair if the status of the looser (represented by their minimum allocation) is not larger than the status of the winner.

It is not so much effort to see that maxmin fairness is a fairness relation. Without a detailed proof, we just give the main reasoning. From $x>_{\text {mmf }} y$ follows that $y>_{m m f} x$ is not possible. From $x>_{m m f} y$ follows $\min \left\{x_{i} \mid x_{i}>y_{i}\right\} \leqslant \min \left\{x_{i} \mid x_{i}<y_{i}\right\}$ and thus $\min \left\{y_{i} \mid y_{i}<x_{i}\right\}<\min \left\{y_{i} \mid y_{i}>x_{i}\right\}$, i.e. the condition for maxmin fairness between $y$ and $x$ is not fulfilled and maxmin fairness is antisymmetric. It requires a little bit formal effort to also see that maxmin fairness is cycle-free, the proof will be presented in a future communication. When reducing the elements of $y$ to another vector $z$ (Pareto-dominated by $y$ ) the new set $A^{\prime}$ for the comparison of $x$ with $z$ will be a superset of $A$ while the new set $C^{\prime}$ will be a subset of $C$. Thus, the inequality $\min \left(A^{\prime}\right) \leqslant \min \left(C^{\prime}\right)$ will still hold and maxmin fairness has the RPT property as well. However, maxmin fairness does not have the LPT property.

(5) Proportional fairness was introduced with regard to an optimization problem of traffic rate allocations, and to overcome some problems with maxmin fairness with regard to maxmin fairness' least element preference. Given a routing for end-to-end user traffic in a wired network with link-capacity constraints (so-called elastic traffic), then user evaluate allocated traffic rates by their utility function, and manipulate traffic rates by payments.

\footnotetext{
${ }^{2}$ In the case that one of these sets is empty, continue the evaluation with $\infty$ instead of min.
} 
The total of utilities minus payments should be maximized. In Kelly (1997) it was shown that generally a price model exists such that the so-called proportional fairness state $x$ can be achieved (and is the one maximizing the objective), where for any other feasible state $y$

$$
I(x, y)=\sum_{i} \frac{y_{i}-x_{i}}{x_{i}} \leqslant 0
$$

holds. This is direct representation of a relation: $x \geqslant_{p f} y$ if and only if $I(x, y) \leqslant 0$. Note that the domain of the relation needs to be $R_{+}^{n}$. From the fact that $x>_{p f} y$ implies $\prod_{i} x_{i}>$ $\prod_{i} y_{i}$ (for the implicator function $I(x, y)=0$ is actually the tangent to the curve $\prod_{i} x_{i}=$ const) follows antisymmetry and cycle-freeness, and RPT can be easily seen from the fact that the indicator function $I(x, y)$ is monotone with regard to the second argument (by anti-monotony with regard to the first argument, also the LPT property follows).

An extension of proportional fairness to $\alpha$-fairness was proposed in Mo and Walrand (2000). Here, the indicator function changes to

$$
I_{\alpha}(x, y)=\sum_{i} \frac{y_{i}-x_{i}}{x_{i}^{\alpha}}
$$

for some $\alpha>0$. For $\alpha \rightarrow \infty \alpha$-fairness approximates maxmin fairness. Also here, $x>_{\alpha} y$ implies a corresponding $>$-relation between the $(1-\alpha)$ power means of the corr. elements, and directly gives antisymmetry and cycle-freeness, while RPT can be directly seen from monotony of the indicator function with regard to the second element.

(6) Leximin relation is another fundamental ordering relation. If the subscript notation $(i)$ indicates the $i$-th smallest element of an ordered set of values, then we compare two vectors $x$ and $y$ by the smallest $j$ such that $x_{(j)} \neq y_{(j)}$. It is said that $x$ leximin dominates $y\left(x \geqslant_{\operatorname{lm}} y\right)$ if either $x=y$ or $x_{(j)}>y_{(j)}$. The relation is transitive and thus cyclefree, also proving antisymmetry and LPT is simple. It is also a complete multi-variate relation.

An important property of all fairness relations is the fact that Pareto-dominance implies fairness. This can be easy seen: if $x \geqslant_{p} y$ then RPT property and antisymmetry give that from $x \geqslant_{R} x$ and $x \geqslant_{p} y$ also $x \geqslant_{R} y$ follows. Thus, as a general result, the maximum set of a fairness relation is a subset of the maximum set of the Pareto-dominance relation. Due to cycle-freeness, this maximum set is never empty (for finite sets). So, a fairness relation can also be seen as the decision maker in multi-objective optimization, excluding the border elements of the Pareto front, where only a few objectives are maximal, other objectives minimal.

There are two limitations that have to be taken into account when using fairness relations for decision making and efficient state selection. (1) The components of the vector need to be commensurable. They should not have different "semantic" meaning (like component 1 being a delay, component 2 a traffic rate) since fairness relations usually also compare between components of a vector. If their components represent different modalities, then we either need to introduce utilities (equity, commodity), which can become a daunting task, or we might use multi-vectors to represent all aspects. (2) Then, maximum 
sets of fairness relations can contain more than one element. If the question about a final decision comes up, all the elements in the maximum set are indifferent with regard to the chosen fairness criterion. Nevertheless, the choice according to global optimality (for example the state with maximal sum of elements) can still be done, so it still can be a rather simple task.

\section{Parabolic Fairness: From Fair Routing to Fair Wireless Channel Allocation}

In this section, we want to demonstrate the concept of universal rationalizability to define a fairness concept for wireless network control. The starting point will be a wired network, where we know about an algorithm (BFC: Bottleneck Flow Control) commonly understood to allocate traffic rates in a fair manner. Then, we use a fairness relation to characterize the final state of the algorithm as the maximum set of that fairness relation. So, we can use the same fairness relation in the context of wireless networks (where the task becomes of discrete nature), without thinking about the transition of the algorithm itself (for example, there is no need to introduce a concept of "bottlenecks"). Since there are several relations able to represent the final state of the Bottleneck Flow Control, we will presents a few results of Monte Carlo simulations for comparing the maximum sets for these relations.

\subsection{Bottleneck Flow Control}

We assume a traffic network congestion avoidance problem. A network for carrying traffic is given as an un-directed graph $G$ with nodes $N$ and links $L$. Also, a maximum capacity is assigned to each link. Then, there are a number of users that want to send traffic units through this network. Thus, also a set of $n$ triples $\left(\Pi_{i}, s_{i}, r_{i}\right)$ of sender-receiver pairs and paths connecting them is given, where $s_{i}$ represents the sending node, $r_{i}$ the receiver node for user $u_{i}$. Paths $\Pi_{i}$ are given as a sequence of joined links starting from $s_{i}$ and ending at $r_{i}$. We also consider the union of all links $l_{i}$ used by all paths, each link with a multiplicity $w_{i}$ according to the number of paths using the link $l_{i}$. Then the Bottleneck Flow Control algorithm, which was initially proposed in Jaffe (1981) assigns traffic amounts $t_{i}$ to all users $u_{i}$ in the following way:

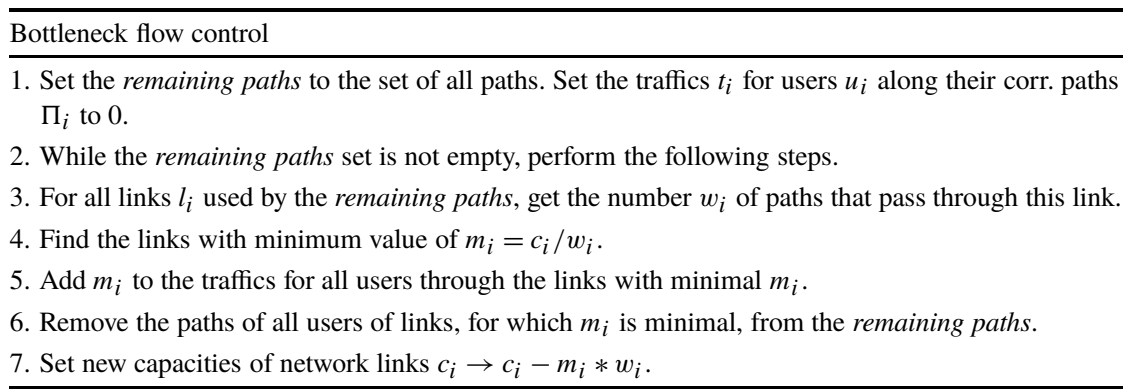


We consider a simple example for the BFC algorithm. Given a network with 7 nodes and links between these nodes as indicated in the figure. It is assumed that the link connecting nodes 3 and 4 has a maximum capacity of 200 units, and the link connecting nodes 4 and 7 has a maximum capacity of 100 units. All other links will have some higher maximum capacity. Three users wants to send traffic through this network: user 1 sends traffic $t_{1}$ via the nodes $1,3,4$ and 7; user 2 sends traffic $t_{2}$ via the nodes 2, 4 and 7; and user 3 sends traffic $t_{3}$ via 5, 3, 4 and 6. It means that some users have to share links for their traffic: users 1 and 3 share the link between nodes 3 and 4, and users 1 and 2 share the link between nodes 4 and 7. The BFC algorithm now will assign specific values for the traffic amounts $t_{1}, t_{2}$ and $t_{3}$, starting with amount 0 , and increasing equally for a subgroup of users. At the beginning (Level 0) the amount will increase for all users. In some later stage, for example at Level 20, the traffic amount assignment of 20 (units) to all users is still feasible. However, at Level 50, the link between nodes 4 and 7 has to transport a total traffic of 100: 50 from user 1, and 50 from user 2. This gives a so-called bottleneck. Any attempt to further increase the traffic for either user 1 or user 2 will result in exceeding the maximum capacity of this link. Thus, the BFC algorithm stops to further increase the traffic amount for users 1 and 2 , and fixes the assignment $t_{1}=50$ and $t_{2}=50$.

However, user 3 is not affected by this bottleneck, since her traffic is not using the link between nodes 4 and 7. So, the BFC algorithm continues. Later on, for example at Level 70, the traffic assignment $t_{3}=70$ (while keeping $t_{1}=t_{2}=50$ ) is still feasible. This level increase will continue until Level 150. Now, the total traffic for the link between nodes 3 and 4 becomes 200: user 1 was fixed before at the level $t_{1}=50$, and $t_{3}=150$ for user 3.

Any further increase of traffic is not possible, and the BFC algorithm stops. Thus, the final traffic assignment is $t_{1}=50, t_{2}=50, t_{3}=150$. In the implementation of this algorithm, of course, there are no increasing level sets, as the values of bottlenecks can be directly inferred from the network configuration and the values of maximum capacities.

Given an instance of a network routing problem with maximum capacities, the BFC algorithm will always assign a unique traffic state to all users. It can be seen that this state corresponds with the maximum set of maxmin fairness, as well as leximin fairness where in both cases, the maximum sets contain exactly one element, and this element is also the single greatest element for these relations (of course, this only holds in the linkcapacity constrained feasible space, i.e. a space with linear inequality constraints). But then, the question comes up if there is also a scalar function of the traffic amounts that is directly maximized by the BFC algorithm. This was shown to be true in Koeppen et al. (2011a). We define a special case of the ordered weighted averaging (OWA) operator. As a reminder, the OWA of a point $x$ of $R^{n}$, given a weight vector $w \in R^{n}$, is defined as $\sum w_{i} x_{(i)}$. In this expression, $x_{(i)}$ indicates the $i$-th smallest element of all coordinates of $x$. We specialize the OWA by also requiring the weights to be sorted in the opposite order: 
Definition 6. Given a point $x$ from $R^{n}$ and a set of weights $w \in R^{n}$, the Ordered-Ordered Weighted Averaging (OOWA) of $x$ by $w$ is defined as

$$
O O W A_{w}(x)=\sum_{i=1}^{n} w_{(i)} x_{(n-i+1)} .
$$

Thus, in the OOWA, the largest value is multiplied with the smallest weight, the second-largest value with the second-smallest weight etc. As a special case of the OOWA, we also introduce the exponential OOWA. The additional requirement here is $w_{(k)}>$ $\sum_{i=1}^{k-1} w_{(i)}$, so that the weights itself are exponentially increasing. A possible choice is $w_{i}=2^{(i-1)}$ for any $i \in N$.

Based on this, in Koeppen et al. (2011a) it was shown:

Theorem 1. Given a weighted graph $G$ of a network, a routing (i.e. a set of linking paths between nodes), then among all feasible traffic allocations to the users, the BFC algorithm gives the state with the maximum value of the exponential OOWA (for any fixed choice of weights).

We also recall two extensions of the result (Koeppen et al., 2011d). The requirement of an exponential increase of the weights is a result of link sharing. The worst case, where the condition of exponential weight increase has to be fulfilled, is where a single user shares traffic with all other users. If there is no link sharing at all, any OOWA will be maximized by the BFC algorithm. So we also consider a linear OOWA with weight-growth law $w_{i}>w_{i-1}$ for any $i>1$. In case that its maximum coincides with the maximum for the exponential OOWA, we can see this as an indication that "link sharing" does not occur, or only to a small degree with low influence on related results. This is a statement without direct reference to the traffic rate allocation problem.

If a link never shares traffic with more than one other user, the requirement of exponential increase of the weights can be relaxed to the requirement $w_{i}>w_{i-1}+w_{i-2}$ for $i>2$ and $w_{2}>w_{1}$. One possible choice for such weights is $w_{i}=F_{i+2}-1$, where $F_{i}$ is the $i$-th element of the Fibonacci series $\left(F_{1}=1, F_{2}=1\right.$ and for $\left.i>3 F_{i}=F_{i-1}+F_{i-2}\right)$. From $w_{i-1}+w_{i-2}=F_{i+1}+F_{i}-2=F_{i+2}-2=w_{i}-1<w_{i}$ it can be seen that this choice fulfills the relaxed weight increase requirement. We will call an OOWA computed with these weights a Fibonacci OOWA. The exposition can be extended by using more than two weights in a straightforward manner. We consider such special versions of the OOWA as a convenient tool for probing implicit sharing issues in any domain, where these expressions can be computed.

For convenience, in the following we will also write expOOWA (for "exponential OOWA"), linOOWA (for "linear OOWA"), FibOOWA (for "Fibonacci OOWA") for these operators with the fixed choice of weights $w_{i}=2^{i-1}$ for the exponential OOWA, $w_{i}=i$ for the linear OOWA, and $w_{i}=F_{i+2}-1$ for the Fibonacci OOWA, and introduce relations $\geqslant_{e o}, \geqslant_{l o}$ and $\geqslant_{f o}$ by size comparison of the corresponding operator values. Since the OWA operator in general is monotone, all three relations are also fairness relations. 


\subsection{Wireless Channel Allocation}

Now we want to turn our attention to a completely different domain, and demonstrate how we can establish a concept of fairness there as well. In Wireless Channel Allocation (WCA), a blank matrix $B$ of channel-timeslot pairs with a total of $M$ cells $b_{i}$, a set $U=$ $\left(u_{i}\right)$ of $N$ user and an $M \times N$ matrix $C$ of channel coefficients of real values from [0,1] are given. The task is to enter at most one user into each blank cell in $B$, i.e. to provide an allocation $a: B \rightarrow U$ of cells to user with $|\{u \in U \mid a(b)=u\}| \leqslant 1$ for all cells $b \in B$. Each entry $c_{i j}$ of the matrix $C$ represents the utility for user $u_{i}$ in case of assignment of cell $b_{j}$, as a model abstraction of all the physical and logistic circumstances of the wireless access. For a given allocation $a$, the performance for each user is given by

$$
p\left(u_{i}\right)=\sum_{j, a\left(b_{j}\right)=u_{i}} c_{i j}
$$

i.e. the sum of channel coefficients for all channels allocated to the user. Channel allocation has to be performed such that, in some sense, all user are "satisfied" with their individual performances as good as possible. The actual problem is to specify the meaning of "satisfied" in an efficient way. For example, considering maximization of the sum of all performances is not a good way to satisfy all users: the optimization problem could be easily solved by selecting for each cell one of the user with maximum channel coefficient. But this way it can happen that then some user will never get any channel allocated, and these users are exempted from wireless access. Therefore, the economics of WCA becomes relevant, especially aspects of fairness.

So the approach is to use expOOWA maximization for the WCA allocation. We have seen that this is indistinguishable from maxmin fairness in fair traffic rate allocation, so it is a "valid" concept for fairness as well, with the additional advantage of being a functional that can be directly computed from performance vectors and do not need pairwise comparisons. Parabolic fairness then is the transfer of this concept of fairness to other domains in the sense of an analogy, as the literature style of a parable does, by imposing expOOWA maximization as the means of efficient state allocation. Then, if we consider the user performance vector, we can introduce a concept of maxmin fairness in the WCA problem as well.

As a small demonstration, we will utilize four relations to random settings of the WCA problem (i.e. all channel coefficients are uniform random samples from [0, 1]) . We select the maximum set $M_{m m f}$ of the maxmin fairness relation, where the relation domain are all possible allocations of users to cells, and compare with the allocations $m_{e o}$ with maximal expOOWA value. Since the number of allocations is growing exponentially, we can do this for small problem scales only. For such problem scales, results are shown in Table 1.

It can be seen that the maximum sets of the maxmin fairness relations are comparable with the number of cells, and that the chance that these maximal elements also include the maximum expOOWA state are increasing with increasing problem scale (with one exception). We can extrapolate that the chances are more than $90 \%$ for realistic problem scales. Table 1 also shows the comparison between the maximum states of expOOWA, linOOWA, 
Table 1

Comparison of maximum set selections by the different relations.

\begin{tabular}{lllll}
\hline$(u, c)$ & $\left|M_{m m f}\right|,($ Quant. $)$ & $m_{e o} \in M_{m m f} ?$ & $m_{l o}=m_{e o} ?$ & $m_{f o}=m_{e o} ?$ \\
\hline$(2,3)$ & $1.73(1,2)$ & 0.53 & 1.0 & 1.0 \\
$(2,4)$ & $1.6(1,2)$ & 0.7 & 1.0 & 1.0 \\
$(2,5)$ & $1.87(2,2)$ & 0.8 & 1.0 & 1.0 \\
$(3,4)$ & $2.6(2,3)$ & 0.63 & 0.83 & 1.0 \\
$(3,5)$ & $2.93(2,3)$ & 0.53 & 0.87 & 1.0 \\
$(4,5)$ & $3.37(2,4)$ & 0.87 & 0.7 & 1.0 \\
\hline
\end{tabular}

For space reasons, column titles are abbreviated: column 1 shows the scale of the problem, i.e. number of user and number of cells; column 2 the average size of the maximum set of maxmin fairness, along with $25 \%$ and $75 \%$ quantiles; column 3 the probability that the allocation with maximal expOOWA is contained in the maximum set of maxmin fairness; columns 4 and 5 then the probabilities that maximum allocation for linOOWA and FibOOWA resp. are equal to the maximum allocation for expOOWA. All values where computed from 30 random samples of the channel coefficients.

and FibOOWA. This allows for a judgement of the "sharing" aspects in this distribution problem, and implicit "bottlenecks." We can see that pairwise sharing is present, since linOOWA and expOOWA differ for larger problem scales, but FibOOWA and expOOWA did not differ in any case in the sample. Remember that for the BFC algorithm final state, the maximum linOOWA would be equal to the maximum expOOWA if there would be no link sharing, and expOOWA and FibOOWA would be equal if there would be at most pairwise link sharing. So we can consider a similar reasoning here, and see that in the WCA problem domain, increased performance for one user will usually need to reduce performance for at most one other user. In WCA, head-to-head competition prevails.

\section{Conclusions and Related Work}

In this paper, a relational framework for utilizing fairness in different domains was introduced. Also a small number of fairness relations have been introduced, but not so much was said with regard to two other main questions. We will shortly comment on them and provide some references.

(1) Are there other fairness relations? The answer is definitely Yes. We may consider a few particular aspects of the fairness relations considered here (esp. maxmin fairness and proportional fairness) in order to see generic aspects. For example, while maxmin fairness is relating different components of the same vector to each other, this is not the case for proportional fairness. It might be seen as an disadvantage, and in fact, proportional fairness can be achieved with arbitrary small components, as long as they are compensated by increasing other components. To achieve a bounded trade-off for proportional fairness, ordered proportional fairness was introduced in Koeppen et al. (2011c). There, the indicator expression changes to

$$
I(x, y)=\sum_{i=1}^{n} \frac{y_{(i)}-x_{(i)}}{x_{(i)}} \leqslant 0
$$


(remember that the subscript $(i)$ indicates the $i$-th smallest component). It can be shown that this is a fairness relation as well.

(2) How can we find the maximum sets of fairness relations? This is in fact a hard question. Even for discrete problems like the WCA, the size of feasible spaces grows exponentially, and exhaustive searches are impossible. This is worsened by the fact that tests for maximum sets needs pairwise comparison, as soon as the relation is not just the comparison of functional values (as for parabolic fairness). So, this is the order of magnitude of the square of feasible space sizes. On the other hand, exact algorithms like Bottleneck Flow Control are not (yet?) known. This gives a good reason to consider the use of metaheuristic approaches. It has happened that over the last few years, a large number of algorithms have been developed to approximate Pareto fronts of multi-objective optimization (MO) problems. The main structural design aspect here is to employ Pareto-dominance instead of direct numerical comparisons (e.g. of fitness, cost, or quality function values). Thus, there is already the laid foundation to impute relations into the algorithmic framework at all. In Koeppen et al. (2010) several meta-heuristic MO algorithms, modified by replacing the role of Pareto-dominance by maxmin fairness, have been compared, and especially the SPEA2 algorithm (Zitzler et al., 2002) appeared to be a good candidate algorithm to handle maximum set approximations in general. However, issues of the relations itself also play a role, and the same algorithm can show strongly differing performances when using different relations. Moreover, in Koeppen et al. (2011b) it was shown that the search for a good relational optimizer is not futile, as the No Free Lunch theorems do not apply to the case of such relational optimization.

\subsection{Related Work}

This work is not the first to consider a formalization ("computable") fairness, and it will not be the last. In fact, the majority of papers evaluating fairness, for example in network simulations, is referring to Jain's fairness indicator (or index) (Jain et al., 1984), computed as

$$
J(x)=\frac{\left(\sum_{i=1}^{n} x_{i}\right)^{2}}{n \cdot \sum_{i=1}^{n} x_{i}^{2}}
$$

This is essentially a measure how strong the components of vector $x$ deviate from equality. Very similar is the Gini index (Gini, 1997). The discussion whether equality is the only acceptable fairness, as it ensures envy-freeness, for example, is still open. It might be so in simple circumstances. As soon as users justify their benefits differently, equality would start to become unfair. This is the main idea of equity. But the main problem with the indicator approach might be seen in the fact that it allows to assign an absolute eternal level of fairness to any vector. As said before, fairness is a matter of comparison, and a state appearing fair in a context of states might appear unfair, if the set of context state changes and new knowledge comes in. There is no way of modeling such a situation with a numerical value alone. 
This argument seems to be in conflict with the parabolic fairness that was introduced before - also here, a numerical value is computed. But by virtue of usage and definition, this expOOWA cannot be considered as a fairness indicator, as its numerical size will strongly depend on the WCA problem at hand. Thus, the only means of using the function value is to compare it with other function values for the same set of channel coefficients. Moreover, the choice of weights is not unique and just needs to follow a weight growth rule.

The same as for fairness indicators can be said about fairness measures (Lan et al., 2010). Also here, some sets of axioms have been provided, with more or less good intentions. Here, we have substantially extended such concepts by basing fairness on comparability of states beyond numbers. In fact, the relational framework allows even symbolic states to be processed, where numeric computations are not possible at all.

As a final comment, much needs to be done with regard to fairness relations and relational optimization. Besides practical and application aspects, also the mathematical foundations need polishing. A main problem is the relation of fairness to topology, i.e. if and how we can consider a system of open neighborhoods of relations, in order to handle limits of relations and other convergence and approximation aspects in a rigid framework. This is one of the topics of ongoing research.

\section{References}

Azar A., Darvishi, Z.A. (2011). Development and validation of a measure of justice perception in the frame of fairness theory - fuzzy approach. Expert Systems with Applications, 38, 7364-7372.

Bertsekas, D., Gallager, R. (1992). Data Networks. Prentice Hall, Englewood Cliffs, 1992

Gini, C. (1997). Concentration and dependency ratios (english translation). Rivista di Politica Economica, 87, 769-789.

Jaffe, J.M. (1981). Bottleneck flow control. IEEE Transactions on Communication, 29(7), 954-962.

Jain, R., Chiu, D.M., Hawe, W. (1984). A quantitative measure of fairness and discrimination for resource allocation in shared computer systems. DEC research report TR-301.

Hack, A., Lammers, F. (2007). On the relation between distributive, procedural, and interactional fairness: evidence from ultimatum games. In: Proceedings of VIII Symposium Zur Ökonomischen Analyse Der Unternehmung.

Kelly, F. (1997). Charging and rate control for elastic traffic. European Transactions on Telecommunications, 8, 33-37.

Köppen, M., Verschae, R., Yoshida, K., Tsuru M. (2010). Comparison of evolutionary multi-objective optimization algorithms for the utilization of fairness in network control. In: Proceedings of 2010 IEEE International Conference on Systems, Man, and Cybernetics (SMC 2010), Istanbul, Turkey, October 2010, pp. 2647-2655.

Köppen, M., Okamoto, J., Honda, A. (2011a). Fuzzy fusion fairness relations for the evaluation of user preference. In: Proceedings of IEEE International Conference on Fuzzy Systems (FUZZ-IEEE 2011), Taipei, Taiwan, June 2011, pp. 2566-2574.

Köppen, M., Yoshida, K., Ohnishi, K. (2011b). A gratis theorem for relational optimization. In: Proceedings 11th International Conference on Hybrid Intelligent Systems (HIS 2011), Melaka, Malaysia, December 2011, pp. 674-679.

Köppen, M., Yoshida, K., Tsuru, M. (2011c). Unsorting the proportional fairness relation. In: Proceedings Third International Conference on Intelligent Networking and Collaborative Systems (INCoS 2011), Fukuoka, Japan, November 2011, pp. 47-52.

Köppen, M., Yoshida, K., Tsuru, M., Oie, Y. (2011d). Annealing heuristic for fair wireless channel allocation by exponential ordered-ordered weighted averaging operator maximization. In: Proceedings of 2011 IEEE/IPSJ International Symposium on Applications and the Internet (SAINT 2011), Munich, Germany, July 2011, pp. 538-543. 
Lan, T., Kao, D., Mung, C., Sabharwal, A. (2010). An axiomatic theory of fairness in network resource allocation. In: INFOCOM 2010, Proceedings IEEE, pp. 1-9.

Mo, J., Walrand, J. (2000). Fair end-to-end window-based congestion control. IEEE/ACM Transactions on Networking, 556-567.

Suzumura, K. (2009). Rational Choice, Collective Decisions, and Social Welfare. Cambridge University Press, Cambridge.

Watanabe, S. (1969). Knowing and Guessing: A Quantitative Study of Inference and Information. Wiley, New York.

Zhang, D., Wang, Y., Lu, J. (2009). On QoS-Guaranteed Downlink Cooperative OFDMA systems with amplifyand-forward relays: Optimal schedule and resource allocation. In: 2009 IEEE Wireless Communications and Networking Conference, April 2009, pp. 1-5.

Zitzler, E., Laumanns, M., Thiele, L. (2002). SPEA2: Improving the strength pareto evolutionary aLgorithm. In: Giannakoglou, K., Tsahalis, D., Periaux, J., Papailou, P., Fogarty, T. (Eds.), EUROGEN 2001, Evolutionary Methods for Design, Optimization and Control with Applications to Industrial Problems, Athens, Greece, 2002, pp. 95-100.

M. Köppen studied physics at the Humboldt-University of Berlin and received his master degree in solid state physics in 1991. Afterwards, he worked as scientific assistant at the Central Institute for Cybernetics and Information Processing in Berlin and changed his main research interests to image processing and neural networks. From 1992 to 2006, he was working with the Fraunhofer Institute for Production Systems and Design Technology. He continued his works on the industrial applications of image processing, pattern recognition, and soft computing, esp. evolutionary computation. During this period, he achieved the doctoral degree at the Technical University Berlin with his thesis works with honors. He has published around 150 peer-reviewed papers in conference proceedings, journals and books and was active in the organization of various conferences as chair or member of the program committee, incl. the WSC on-line conference series on Soft Computing in Industrial Applications, and the HIS conference series on Hybrid Intelligent Systems. He is founding member of the World Federation of Soft Computing, and also member of the editorial board of the Applied Soft Computing journal, the Intl. Journal on Hybrid Intelligent Systems and the Intl. Journal on Computational Intelligence Reserach. In 2006, he became JSPS fellow at the Kyushu Institute of Technology in Japan, and in 2008 Professor at the Network Design and Reserach Center (NDRC) of the Kyushu Institute of Technology, where he is conducting now research in the fields of multi-objective optimization, digital convergence and multimodal content management. 


\title{
Sąryšinis optimizavimas ir jo taikymai: nuo srauto siaurame praėjime valdymo iki belaidžio ryšio kanalų paskirstymo
}

\author{
Mario KÖPPEN
}

Sąryšių matematika, kuri yra studijuojama tokiose disciplinose kaip matematinè ekonomika ir socialinio pasirinkimo teorija, suteikia turtingus ir bendrus principus bei atrodo natūralus ir tiesioginis būdas performuluoti optimizavimo tikslus, aprašyti vartotojų pageidavimus, pagrịsti sąžiningumo kriterijus, vertinti paslaugų kokybę ar naudą. Čia mes sutelkiame demesị i konkrečius formaliujų sąryšių taikymo aspektus tinklų projektavimo ir valdymo uždaviniuose bei pateikiame bendrą sąryšinio optimizavimo idejją. Sąryšiniame optimizavime uždavinys aprašomas formaliuoju sąryšiu, o sprendinys maksimalių (arba nedominuojamų) sąryšio elementų aibe. Tai atrodo natūraliu standartinio optimizavimo išplètimu, ir taip pat apima kitas optimumo sąvokas. Kartu mes pateiksime teisingumo sąryšių, kurie gali būti naudojami kaip maksimizuojantys sąryšiai pagal įvairius taikymo poreikius, aibę ir aprašysime maksimumų aibių aproksimavimo metaeuristinį metodą, išvestą iš evoliucinių daugiakriterio optimizavimo algoritmų. 